\title{
Kübik AlLiSi yapıdaki XMnSb (X=Au ve Ir) bileşiklerinin yapısal, mekanik ve dinamik özellikleri
}

\author{
Selgin $\mathrm{AL}^{*, 1}$, Nihat ARIKAN ${ }^{2}$ \\ ${ }^{1}$ Ahi Evran Üniversitesi, Fen Edebiyat Fakültesi, Fizik Bölümü, Kırşehir \\ ${ }^{2}$ Ahi Evran Üniversitesi, Eğitim Fakültesi, Matematik ve Fen Bilgisi Eğitimi Bölümü, Kırşehir \\ Gelis Tarihi (Recived Date): 15.08.2017 \\ Kabul Tarihi (Accepted Date): 17.10 .2017
}

\begin{abstract}
Özet
Kübik AlLiSi yapıdaki XMnSb ( $X=A u$ ve Ir) bileşiklerinin yapısal, elektronik, elastik ve fonon özellikleri ilk prensip hesaplamalarıyla gerçekleştirildi. Optimize örgü sabiti ve hacim modülü hesaplandl ve mevcut verilerle karş̧laş̧tırıldı. Kübik AlLiSi yapıdaki XMnSb $\left(X=A\right.$ u ve Ir) bileşiklerinin elastik sabitleri $\left(C_{11}, C_{12}\right.$ ve $C_{44}$ ) enerji zorlama metodu kullanilarak hesapland. AuMnSb ve IrMnSb bileşiklerinin spin polarize bant yapıları spin yukarı ve spin aşağı eşleşmeleri için hesaplanarak mevcut verilerle karş̧ılaştırıldı. Fonon dağıllım eğrileri ve onlara karşıllk gelen durum yoğunlukları yoğunluk fonksiyonel pertürbasyon teorisi çerçevesinde lineer tepki yaklaşımı kullanılarak ilk defa elde edildi.
\end{abstract}

Anahtar Kelimeler: Bant yapısı, yoğunluk fonksiyonel teorisi, elastik sabiti, fonon.

\section{Structural, mechanical and dynamical properties of $\mathrm{XMnSb}(\mathrm{X}=\mathrm{Au}$ and $\mathrm{Ir})$ in the AlLiSi structure}

\begin{abstract}
First principle calculations have been carried out on the structural, electronic, elastic, and phonon properties of XMnSb (X $=A u$ and Ir) in the cubic AlLiSi structure. The optimized lattice constant and bulk modulus are calculated and compared with the available data. The elastic constants $\left(C_{11}, C_{12}\right.$ and $\left.C_{44}\right)$ in the cubic AlLiSi structure for XMnSb ( $X=A u$ and Ir) are computed using the energy-strain method. Spin polarized band structures of $\mathrm{AuMnSb}$ and IrMnSb for majority-spin and minority spin alignments are calculated and analyzed in comparison with the existing findings. Phonon dispersion curves and their corresponding total and projected densities of states have been obtained for the first time using a linear response approximation in the framework of the density functional perturbation theory.
\end{abstract}

Keywords: Band structure, density functional theory, elastic constant, phonon.

\footnotetext{
* Selgin AL, selgin.al@ahievran.edu.tr, http://orcid.org/0000-0003-2496-1300

Nihat ARIKAN, narikan@ ahievran.edu.tr, http://orcid.org/0000-0001-8028-3132
} 


\section{Giriş}

Üçlü Heusler alaşımlarının iki ayrı ailesi vardır; bunlardan biri $L 2_{l}$ yapısında kristalleşen tam Heusler alaşımları olup $\mathrm{X}_{2} \mathrm{YZ}$ formülüne sahiptir [1]. İkinci aile ise yarı Heusler alaşımlardır, genel formülü XYZ olup AlLiSi $\left(C l_{b}\right)$ yapıda kristalleşirler [2-4] . Burada, $X$ tipik bir ağır geçiş metalidir, $\mathrm{Y}$ bir geçiş metali veya bir nadir toprak metali iken, $\mathrm{Z}$ bir ana grup III, IV veya $\mathrm{V}$ elemanıdır. $L 2_{l}$ ve $C l_{b}$ yapılarında kristalleşen mangan tabanlı üçlü intermetallik alaşımlar, metalik spin majority bant ve yarı iletken minority spin bantlar tarafindan karakterize edilen yarı-metalik ferromagnetler gibi zengin elektronik çeşitlilik gösterirler. Mangan-tabanlı Heusler alaşımlar $(\mathrm{XMnSb})$ potansiyel teknolojik uygulamalar bakımından ferromanyetik materyallerin temel fiziksel özelliklerine sahiptir. AuMnSb ve aynı sınıftan diğer bazı yarı Heusler alaşımlarının elektrik direnci ve hall effect değerlerini Otto ve ark. [4] tarafındanrapor edildi.. AuMnSb alaşımının kristal yapısı, mikro yapısı ve manyetik özellikleri de Otto ve ark. [3] tarafından incelendi. Bu alaşımın manyetik özelliklerinin, $0 \mathrm{~K}^{\prime}$ de doyum momentine karşılık gelen değerden farklı etkili bir paramanyetik moment gösterdiğini önerdiler. AuMnSb ve AuMnSn alaşımlarının elektronik bant yapıları Offernes ve ark. [2, 5, 6] tarafından çalışıldı. AuMnSb sistemindeki faz ilişskileri, toz $X$ ışın kırınımı, metalografi, elektron mikroprob analizi ve termal analiz ile Walle ve ark. [7] tarafindan çalışılmıştır. Yarı Heusler alaşımlarından AuMnX (X = In, Sn, Sb) 'nin ferromanyetik taban durumları, yerel spin yoğunluk yaklaştırması (LSDA) ve yoğunluk fonksiyonel teorisine (DFT) göre Amft ve Oppeneer [8] tarafından hesaplandı. AuMnSn, yarı metalik bir ferromagnet olarak hesaplanırken, 'AuMnSb ve AuMnIn' nin farklı bant doluluklarından dolayı yarı metalik olmadığını önerdiler. Mn tabanlı yarı Heusler alaşımların, $X Y Z$ 'nin $(X=I r, P t, A u, Y, M n, Z=S n, S b)$ yapisal faz kararlılı̆̆ manyetik özellikleri yoğunluk fonksiyonel teorisi kullanılarak Amudhavalli ve ark. [9] tarafından da analiz edilmiştir. IrMnSb alaşımının yapısal, elektronik ve manyetik özellikleri çeşitli araştırma grupları tarafından çalışılmıştır [2, 5, 9-17] . Çeşitli teorik metotlarla bazı araştırma grupları $[10,12,13$, $15,17] \mathrm{IrMnSb}$ alaşımının spin polarize elektronik bant yapisinı hesapladılar. FP LAPW metot kullanılarak IrMnZ ( $\mathrm{Z}=\mathrm{Al}$, Sn ve Sb) yarı Heusler alaşımlarının yapısal ve elastik özellikleri detaylı olarak Hamidani ve ark. [11] tarafından çalışılmıştır.

AuMnSb ve IrMnSb alaşımlarının bazı yapısal ve elektronik özellikleri teorik ve deneysel açıdan incelenmesine rağmen, bu alaşımların dinamik özellikleri yeterli düzeyde incelenmemiştir.
Örneğin, örgü dinamiğini anlamak için tam fonon dağıllım eğrilerini elde etmek gereklidir. Fonon spektrumunun bilinmesi, termodinamik kararlılık, faz geçişi ve termal transport gibi materyal özelliklerini belirlemek için çok önemlidir. $\mathrm{Bu}$ sebeple, bu çalışma lineer tepki yöntemi kullanarak $\mathrm{AuMnSb}$ ve IrMnSb alaşımlarının tam fonon özelliklerini araştırmaya odaklanmaktadır.

\section{Metot}

Hesaplamalar Quantum ESPRESSO paket programı [18] kullanılarak yapıldı. Program, yoğunluk fonksiyonel teori ve düzlem dalga temel setine dayanmaktadır. Elektronik değişim korelasyon potansiyeli, Perdew Burke Ernzerhof (PBE) şeması kullanılarak genelleştirilmiş eğim yaklaşımı (GGA) ile hesapland [19]. Kendinden tutarlı alan (self consistent field) hesaplamasındaki düzlem dalga kesme enerjisi, 40 Ry'ye eşit olarak alındı. Brillouin bölge integrasyonları, 10x10x10 k nokta mesh kullanılarak gerçekleştirildi. Fermi yüzeyine kadar olan integrasyon, smearing tekniği kullanılarak [20] ve smearing parametresi 0.02 Ry alınarak gerçekleştirildi. Kohn Sham denklemlerinin kendi kendine tutarlı çözümlerini elde ettikten sonra, örgü dinamik özellikleri, kendinden tutarlı yoğunluk fonksiyonel pertürbasyon teorisi $[21,22]$ çerçevesinde hesaplanmıştır. Tam fonon dağınım eğrileri ve durum yoğunluğunu elde etmek için, sekiz dinamik matris bir $4 \times 4 \times 4$ q nokta kümesi üzerinde hesaplandı. Bu dinamik matrisler, bu küme üzerinde bir Fourier ters dönüşümü ile değerlendirilebilir. Elastik sabitler toplam enerjinin hacim korumalı zorlamanın bir fonksiyonu olarak hesaplanmıştır. Hacim modülü, $B, C_{44}$ ve kesme modülü $C^{\prime}=\left(C_{11}-C_{12}\right) / 2$, sirasiyla hidrostatik basinçtan (e $=\delta, \delta, \delta, 0,0,0)$, üç boyutlu kesme zorlama (e $=0,0,0, \delta, \delta, \delta)$ ve hacim korumalı ortorombik zorlama $\left(\mathrm{e}=\delta, \delta,(1+\delta)^{-2}-1,0,0,0\right)$ kullanılarak hesaplanmıştır. Burada $B$, aşağıdaki denklemden elde edilmiştir;

$\frac{\Delta E}{V}=\frac{9}{2} B \delta^{2}$

Burada $\mathrm{V}$ zorlanma uygulanmamış örgü hücresinin hacmi, $\Delta E$ ise e $\left(e_{1}, e_{2}, e_{3}, e_{4}, e_{5}, e_{6}\right)$ vektörleriyle uygulanan bir zorlamanın sonucu olarak ortaya çıkan enerji değişimidir. $\quad C^{\prime}$ de aşağıdaki bağlantıdan elde edilebilir;

$\frac{\Delta E}{V}=6 C^{\prime} \delta^{2}+0 \delta^{3}$

Yukarıdaki iki değerlerden $\left(B\right.$ ve $\left.C^{\prime}\right), C_{11}=$ $\left(3 \mathrm{~B}+4 C^{\prime}\right) / 3$ ve $C_{12}=\left(3 \mathrm{~B}-2 C^{\prime}\right) / 3$ elde edilir ve $C_{44}$ de aşağıdaki denklem kullanılarak elde edilir.

$\frac{\Delta E}{V}=\frac{3}{2} C_{44} \delta^{2}$ 
Kübik yapı için kesme $(\mathrm{G})$, Voigt $\left(G_{v}\right)$ ve Reuss $\left(G_{R}\right)$ modülü aşağıdaki gibi tanımlanmıştır;

$$
\begin{aligned}
& G=\frac{G_{v}+G_{R}}{5}, \quad G_{v}=\frac{C_{11}-C_{12}+3 C_{44}}{5} v e \\
& G_{R}=\frac{5 C_{44}}{\left[4 C_{44}+3\left(C_{11}-C_{12}\right)\right]}
\end{aligned}
$$

\section{Poisson oranı aşağıdaki denklemde verilmiştir;}

$$
\sigma=\frac{3 \mathrm{~B}-2 \mathrm{G}}{2(3 \mathrm{~B}+\mathrm{G})}
$$

\section{Bulgular}

Burada mangan tabanlı AuMnSb ve $\mathrm{IrMnSb}$ alaşımları $C l_{b}$ fazında incelenmiştir. AuMnSb ve IrMnSb alaşımlarının $C l_{b}$ fazı AlLiSi yapısında bulunur ve Şekil 1'de görüldüğü gibi F-43m uzay grubu ile kübik bir yapıda kristalleşir. Birim hücredeki atomlar aşağıdaki Wyckoff pozisyonlarına yerleştirilir: $\mathrm{Au}(\mathrm{Ir})=(0,0,0), \mathrm{Mn}=(0.25,0.25$, $0.25)$ ve $\mathrm{Sb}=(0.50,0.50,0.50)$. İlk adım olarak, denge örgü sabitleri, örgü sabitlerinin farklı değerlerine karşılık gelen toplam enerjinin minimize edilmesiyle belirlenmiştir. Ardından, denge örgü sabitini $\left(a_{0}\right)$ ve hacim modülünü $(B)$ elde edebilmek için, toplam enerji hacim verileri Murnaghan durum denklemi [23] kullanılarak elde edildi. AuMnSb ve IrMnSb alaşımları için elde edilen örgü sabiti ve hacim modülü değerleri mevcut deneysel ve teorik verilerle birlikte Tablo 1'de sunulmuştur. Her iki alaşım için elde edilen örgü sabiti değerleri, mevcut deneysel ölçümler $[2,3,5,6,8,12]$ ve teorik sonuçlar ile oldukça iyi uyum içindedir [5, 9-15] . Sırasıyla, AuMnSb ve IrMnSb için tahmin edilen $74.4 \mathrm{GPa}$ ve $112.6 \mathrm{GPa}$ hacim modülü değerleri, VASP metot kullanılarak hesaplanan (110.26 GPa ve $138.78 \mathrm{GPa})$ değerlerden küçüktür. $\mathrm{Bu}$ alaşımlarda hacim modülü örgü parametresindeki azalmayla artmaktadır.

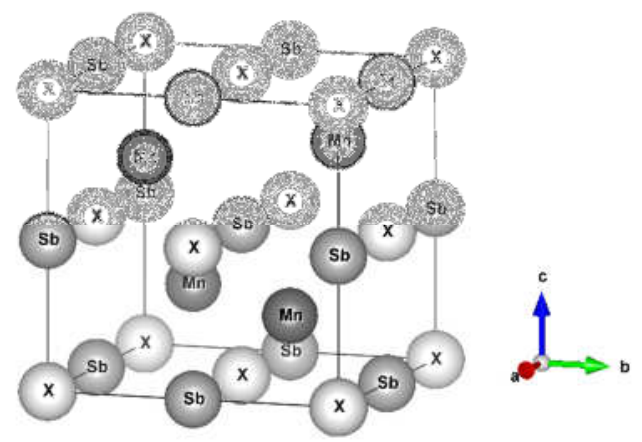

Şekil 1. XMnSb (X=Au ve Ir) için AlLiSi $\left(C 1_{b}\right)$ kristal yapis1.
AuMnSb ve IrMnSb alaşımları için hesaplanan toplam manyetik momentlerin $\left(\mu_{\text {top }}\right)$ hesaplanan değerleri, karşılaştırma için ölçülen deneysel değerler $[3,5,6,8,12]$ ve teorik değerlerle $[5,8,9$, 12-15] birlikte Tablo 1'de verilmektedir. AuMnSb için hesaplanan toplam manyetik moment değeri mevcut deneysel değerlerden yaklaşık \%15 oranında daha küçüktür. Teorik açıdan ise mevcut VASP metot sonuçlarından [9] \%10.63 daha büyük olmakla beraber, bu çalışmada elde edilen sonuç Tablo 1 de verilen deneysel değere daha yakındır. IrMnSb için ise mevcut teorik ve deneysel ölçümlerle oldukça uyum içerisindedir.

Bir kübik kristalin elastik özellikleri, üç bağımsız elastik sabitleri, yani $C_{11}, C_{12}$ ve $C_{44}$ tarafından tanımlanır. $\quad C_{11}$, tek yönlü sıkıştırmaya karşı gösterilen direnç $(<100\rangle$ ana yön boyunca sıkıştırma), $C_{44}, \quad(100)$ düzlemi boyunca [110] yönünde kesilme deformasyonuna karşı ortaya çıkan direnci temsil eder ve $C_{12}$ basitçe fiziksel bir anlam taşımazken, $C_{11}$ ve $C_{44}$ ile kombinasyonu materyallerin elastik davranışı hakkında ek bilgi verir. Örnek olarak, tetragonal kesme sabiti $\mathrm{C}^{\prime}=$ $\left(C_{11^{-}} C_{12}\right) / 2$ [110] yönünde (110) düzlemi boyunca kesme deformasyonuna direnç gösterir. Burada hesaplanan $C_{11}, C_{12}$ ve $C_{44}$ değerleri, karşılaştırma için mevcut teorik bulgular $[9,11]$ ile birlikte Tablo 2'de özetlenmiştir. $\mathrm{Bu}$ alaşımların mekanik kararlılıkları elastik sabitleri açısından analiz edilmiştir. Kübik kristaller için mekanik kararlılık koşulları [24] aşağıda verilmiştir;

$$
C_{44}>0 ;\left(C_{11}-C_{12}\right) / 2>0 \text { ve } B=\left(C_{11}+2 C_{12}\right) / 3>0
$$

Tablo 2'den açıkça görüleceği üzere, bu kriterler doğrulanmış ve böylelikle her iki alaşımın da $C 1_{b}$ fazda mekanik kararlı oldukları görülmüştür. $\mathrm{AuMnSb}$ ve IrMnSb alaşımlarının mekanik özellikleri (kırılganlık ve süneklik) hacim modülünün kesme modülüne oranı $(B / G)$ kullanılarak incelenmiştir. Pugh [25] kriterine göre, $B / G$ oranı 1,75 değerinden küçük ise, materyal kırılgan davranış gösterirken, bu oran 1,75 değerinden büyük ise materyal sünek bir doğaya sahiptir. AuMnSb ve IrMnSb alaşımları için $B / G$ değeri sirasiyla 3.470 ve 2.863 olarak hesaplanmıştır. Buna göre, her iki materyalin da sünek bir doğası vardır ve mevcut teorik veriler [9, 11] bunu desteklemektedir. $C 1_{b}$ fazındaki AuMnSb ve IrMnSb alaşımların elastik sabitleri için deneysel veri mevcut değildir, bu nedenle bu tahmini değerleri deneysel onay beklemektedir. $\mathrm{Bu}$ alaşımlar için Poisson oranları $(\sigma)$ da hesaplanmış olup Tablo 2' de verilmiştir. Kovalent bağlı malzemeler için Poisson oranı $\sigma=0.1$ civarında iken iyonik bağlı malzemeler için bu oran 0.25 civarındadır. Tablo 2' de görüldüğü üzere bu çalışmadaki alaşımlar iyonik karakterdedir ve 
literatürdeki teorik veri $[9,11]$ ile de uyum içerisindedir.

Tablo 1. AuMnSb ve IrMnSb alaşımları için hesaplanan örgü sabitleri ( $)$, hacim modülü (GPa), toplam manyetik moment $\left(\mu_{B}\right)$, hacim modülünün basınç türevi.

\begin{tabular}{|c|c|c|c|c|c|}
\hline Malzeme & Kaynak & $a$ & $B$ & $B^{\prime}$ & $\mu_{B \text { toplam }}$ \\
\hline \multirow[t]{11}{*}{$\mathrm{AuMnSb}$} & Bu çalışma & 6.248 & 74.4 & 4.03 & 3.63 \\
\hline & Deney [3] & 6.373 & & & 5.7 \\
\hline & Deney [2] & 6.379 & & & \\
\hline & Deney [5] & 6.164 & & & 4.2 \\
\hline & FLMTO [5] & -- & & & 4.56 \\
\hline & Deney [7] & 6.379 & & & 4.2 \\
\hline & Deney [8] & 6.379 & & & 4.2 \\
\hline & Teori [8] & 6.197 & & & 4.24 \\
\hline & VASP GGA [9] & 6.121 & 110.26 & 5.12 & 3.281 \\
\hline & FLAPW GGA [12] & 6.448 & & & 4.66 \\
\hline & Deney [12] & 6.377 & & & 5.05 \\
\hline \multirow[t]{12}{*}{$\mathrm{IrMnSb}$} & Bu çalıșma & 5.992 & 112.6 & 4.72 & 3.13 \\
\hline & Deney [2] & 6.164 & & & \\
\hline & Deney [5] & 6.164 & & & 3.1 \\
\hline & TB LMTO [5] & & & & 3.08 \\
\hline & VASP GGA [9] & 5.918 & 138.78 & 5.87 & 2.984 \\
\hline & VASP GGA [10] & 6.114 & & & \\
\hline & KKR LDA [14] & -- & & & 3.126 \\
\hline & FLAPW GGA [12] & 5.929 & & & 3.11 \\
\hline & Deney [12] & 6.164 & & & 3.10 \\
\hline & KKR LDA [15] & -- & & & 3.022 \\
\hline & FSKKR LDA [13] & -- & & & 3.022 \\
\hline & FP LAPW [11] & 6.155 & 128.16 & & \\
\hline
\end{tabular}

Tablo 2. $C 1_{b}$ yapıdaki AuMnSb ve IrMnSb alaşımlarının hacim modülü (GPa), elastik sabitler (GPa), Kayma modülü $(\mathrm{GPa})$ ve $B / G$ oranı.

\begin{tabular}{|c|c|c|c|c|c|c|c|c|c|c|}
\hline Malzeme & Kaynak & $\boldsymbol{B}$ & $C_{11}$ & $C_{12}$ & $C_{44}$ & $\sigma$ & $G$ & $G_{v}$ & $G_{r}$ & $B / G$ \\
\hline \multirow[t]{2}{*}{$\mathrm{AuMnSb}$} & Bu çalışma & 78.019 & 89.195 & 72.430 & 42.413 & 0.36 & 22.48 & 28.80 & 16.16 & 3.470 \\
\hline & VASP GGA [9] & 117.98 & 183.28 & 85.33 & 40.48 & 0.35 & 43.88 & & & 2.688 \\
\hline \multirow[t]{3}{*}{$\mathrm{IrMnSb}$} & Bu çalışma & 118.846 & 149.373 & 103.582 & 61.762 & 0.34 & 41.49 & 46.21 & 36.78 & 2.863 \\
\hline & VASP GGA [9] & 152.32 & 206.02 & 125.48 & 34.33 & 0.40 & 36.70 & & & 4.150 \\
\hline & FP LAPW [11] & 134.13 & 158.39 & 122.00 & 98.34 & 0.38 & 50.94 & & & 2.633 \\
\hline
\end{tabular}

a)
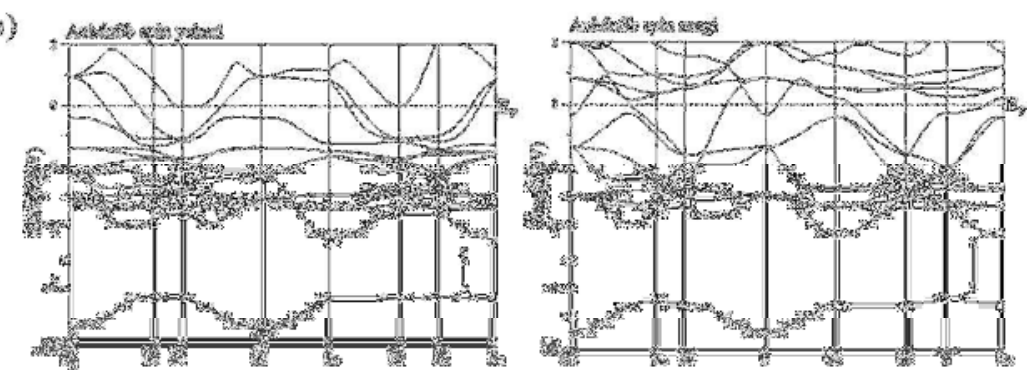

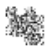
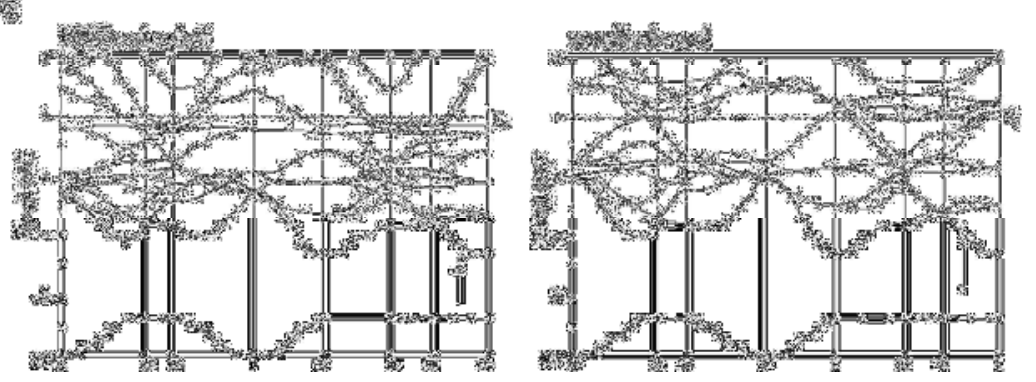

Şekil 2. a) AuMnSb ve b) IrMnSb alaşımlarının spin polarize elektronik bant yapısı (Kesikli çizgiler Fermi seviyesini göstermektedir.) 

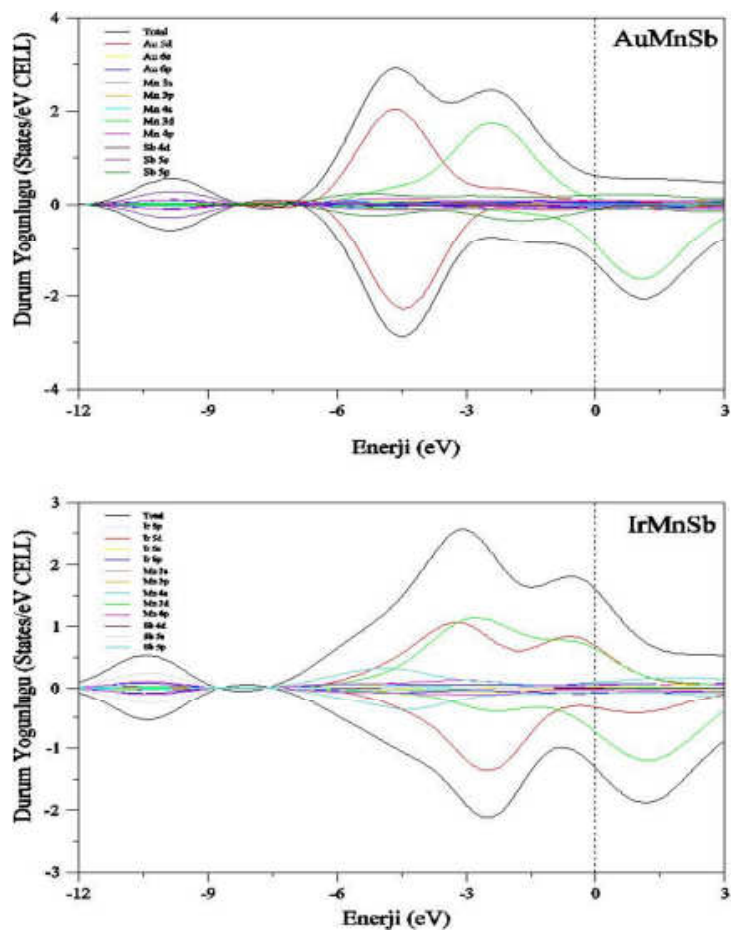

Şekil 3. AuMnSb ve IrMnSb alaşımlarının spine bağlı toplam ve kısmi durum yoğunlukları. (Kesikli çizgiler Fermi seviyesini göstermektedir.)

Spin yukarı ve spin aşağı gösterimleri için AuMnSb ve IrMnSb alaşımlarının spin polarize bant yapıları Şekil 2'de birinci Brilloin bölgesinde yüksek simetri yönleri boyunca sunulmuştur. Şekil 2'den görüldüğü üzere, hem spin yukarı hem de spin aşağı için elektronik bant aralıklarının olmadığı görülmektedir. Her iki alaşım için toplam ve parçalı durum yoğunlukları Şekil 3'de verilmiştir. Durum yoğunluğu grafikleri her iki alaşım içinde Fermi seviyesinde bir enerji aralığı içermemektedir. $\mathrm{Bu}$ durum, spin yukarı ve spin aşağı durumları için polarize bant yapılarının metalik davranış sergilediğini göstermektedir. Her iki alaşım içinde hesaplanan elektronik bant yapıları önceki rapor edilmiş çalışmalarla uyum içerisindedir $[8-10,12$, $13,15]$. Fermi seviyesindeki durum yoğunluğuna baskın katkı AuMnSb için spin yukarı da Sb 5p, spin aşağı için Mn 3d durumlarından kaynaklanmaktadır. IrMnSb için ise hem spin yukarı hemde spin aşağı durumlarına Fermi seviyesine başlıca katkı Ir 5d ve Mn 3d dan gelmektedir. Fermi seviyesinin altındaki bantlar AuMnSb için $\mathrm{Au} 5 \mathrm{~d}$ ve $\mathrm{Mn} 3 \mathrm{~d}$ durumlarından, IrMnSb için ise Ir $5 \mathrm{~d}$ ve $\mathrm{Mn} 3 \mathrm{~d}$ durumlarından kaynaklanmaktadır.

AuMnSb ve IrMnSb alaşımlarının örgü dinamik özellikleri, Quantum ESPRESSO paket programında [18] uygulanan lineer tepki yaklaşımını kullanarak çalışılmıştır. Şekil 4, birinci Brillouin bölgesindeki çeşitli simetri çizgileri boyunca hesaplanan fonon dağılım eğrilerini ve AuMnSb ve IrMnSb için karşılık gelen toplam ve projektif fonon durum yoğunluğu (pDOS) göstermektedir. $\quad C 1_{b}$ yapısının birim hücresi üç atom içerdiğinden, toplam dokuz fonon modu mevcut olup, bunların üçü akustik ve altısı optik dallardan oluşmaktadır. Şekil 4'de görüldüğü gibi, tüm fonon modları pozitif frekanslara sahip olduğundan, bu alaşımlar $C 1_{b}$ fazında dinamik olarak kararlıdır. Her iki alaşımdaki elementler arasında en hafif olanı $\mathrm{Mn}$ atomlarıdır. Bundan dolayı fonon spektrumunda en üst optik frekans bölgesinde $\mathrm{Mn}$ atomlarının titreştiği görülmektedir (Şekil 4 sağ panel). Şekil 4'de görüleceği üzere orta frekans bölgesinde $\mathrm{Sb}$ atomlarının ve en alt akustik frekans bölgesinde ise ağı Au ve Ir atomları titreşmektedir.
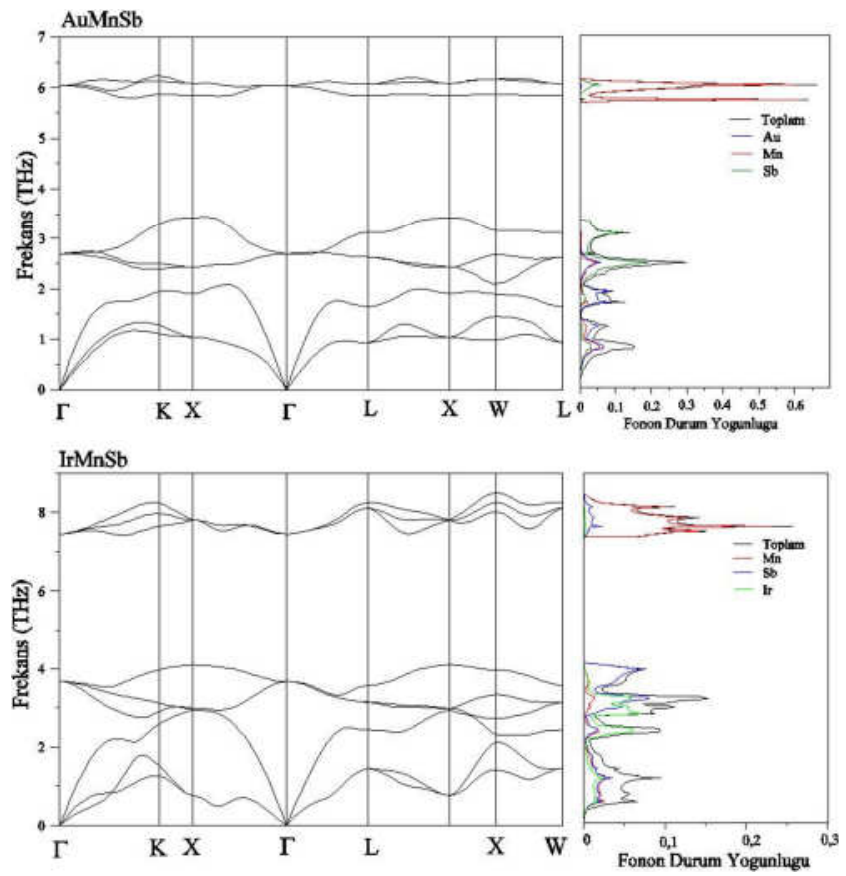

Şekil 4. AuMnSb ve IrMnSb alaşımlarının için hesaplanan fonon dağınım eğrileri ve fonon durum yoğunlukları.

\section{Sonuçlar}

Bu çalışmada, $C 1_{b}$ fazdaki üçlü AuMnSb ve IrMnSb alaşımlarının yapısal, elastik, elektronik ve dinamik özelliklerini incelemek için $a b$ initio hesaplamaları yapılmıştır. Denge örgü parametreleri için elde edilen değerler, teorik ve mevcut deneysel veriler ile iyi bir uyum içindedir. IrMnSb alaşımın bulk modülü AuMnSb alaşımın bulk modülünden daha büyük olduğu için IrMnSb alaşımı daha sert malzemedir. Hesaplanan elastik sabitlerin analizi alaşımların mekanik olarak kararlı olduğunu göstermektedir. Pugh kriterine göre, her iki alaşım da sünek özellik göstermiş olup mevcut verilerle uyum içerisindedir. Alaşımlar için hesaplanan elektronik bant yapıları ve bunlara karşılık gelen toplam ve parçalı durum yoğunlukları ve toplam manyetik moment mevcut verilerle 
karşılaştırılmıştır. Bant yapısı ve durum yoğunluk diyagramları, $C 1_{b}$ fazda AuMnSb ve IrMnSb alaşımlarının metalikliğini teyit etmektedir. $C 1_{b}$ fazındaki AuMnSb ve IrMnSb alaşımları için fonon dağınım eğrileri ve bunlara karşılık gelen toplam ve parçalı durum yoğunluklar. Yoğunluk fonksiyonel pertürbasyon teorisi çerçevesinde ilk defa hesaplanmıştır. Her iki alaşımda negatif fonon

\section{Kaynakça}

[1] Villars, P. and Calvert, L.D., Pearson's handbook of crystallographic data for intermetallic phases (2nd ed). ASM International, Materials Park, OH, (1991).

[2] Offernes, L., Ravindran, P., Seim, C.W. and Kjekshus, A., Prediction of composition for stable half-heusler phases from electronicband-structure analyses, Journal of Alloys and Compounds, 458, 47-60, (2008).

[3] Otto, M.J., van Woerden, R.A.M., van der Valk, P.J., Wijngaard, J., van Bruggen, C.F., Haas, C. and Buschow, K.H.J., Half-metallic ferromagnets. 1. structure and magnetic properties of $\mathrm{NiMnSb}$ and related intermetallic compounds, Journal of Physics: Condensed Matter, 1, 2341, (1989).

[4] Otto, M.J., Feil, H., van Woerden, R.A.M., Wijngaard, J., van der Valk, P.J., Van Bruggen, C.F. and Haas, C., Electronic structure and magnetic, electrical and optical properties of ferromagnetic heusler alloys, Journal of Magnetism and Magnetic Materials, 70, 33-38, (1987).

[5] Offernes, L., Ravindran, P. and Kjekshus, A., Electronic structure and chemical bonding in half-heusler phases, Journal of Alloys and Compounds, 439, 37-54, (2007).

[6] Offernes, L., Ravindran, P. and Kjekshus, A., Prediction of large polar kerr rotation in the heusler-related alloys AuMnSb and AuMnSn, Applied Physics Letters, 82, 2862-2864, (2003).

[7] Walle, C., Offernes, L. and Kjekshus, A., The ternary system $\mathrm{Au}-\mathrm{Mn}-\mathrm{Sb}$ and the AuMnSn1-XSbx phase, Journal of Alloys and Compounds, 349, 105-110, (2003).

[8] Amft, M. and Oppeneer, P.M., Calculated magneto-optical kerr spectra of the halfheusler compounds AuMnx (X = In, Sn, Sb), Journal of Physics: Condensed Matter, 19, 315216, (2007).

[9] Amudhavalli, A., Rajeswarapalanichamy, R. and Iyakutti, K., Structural, electronic, mechanical and magnetic properties of $\mathrm{Mn}$ based ferromagnetic half heusler alloys: A first principles study, Journal of Alloys and Compounds, 708, 1216-1233, (2017). frekansına sahip olmadıkları için dinamik olarak kararlıdır.

\section{Teşekkür}

Bu çalışma, Ahi Evran Üniversitesi bilimsel araştırma projeler birimi, AHILAB. A3.17.002 numaralı projesi, tarafından desteklenmiştir.

[10] Ibrica, T., Juliana, H., Bradley, S., Paul, G., Tula, R.P., Andrei, S., Evgeny, Y. T. and Pavel, V.L., Effects of pressure and strain on spin polarization of IrMnSb, Journal of Physics: Condensed Matter, 29, 075801, (2017).

[11] Hamidani, A., Bennecer, B. and Boutarfa, B., Structural and elastic properties of the halfheusler compounds IrMnZ $(\mathrm{Z}=\mathrm{Al}, \mathrm{Sn}$ and $\mathrm{Sb})$, Materials Chemistry and Physics, 114, 732-735, (2009).

[12] Kulkova, S.E., Eremeev, S.V., Kakeshita, T., Kulkov, S.S. and Rudenski, G.E., The electronic structure and magnetic properties of full- and half-heusler alloys, Materials Transactions, 47, 599-606, (2006).

[13] Galanakis, I., Mavropoulos Ph. and Dederichs, P.H., Electronic structure and Slater-Pauling behaviour in half-metallic heusler alloys calculated from first principles, Journal of Physics D: Applied Physics, 39, 765, (2006).

[14] Galanakis, I., Orbital magnetism in the halfmetallic heusler alloys, Physical Review B, 71, 012413, (2005).

[15] Galanakis, I., Dederichs, P.H. and Papanikolaou, N., Origin and properties of the gap in the half-ferromagnetic heusler alloys, Physical Review B, 66, 134428, (2002).

[16] Brandão, D.E. and Gomes, A.A., A simple picture for the lattice parameter behaviour in heusler C1b compounds, Physica Status Solidi A, 142, 27-33, (1994).

[17] De Groot, R.A. and Buschow, K.H.J., Recent developments in half-metallic magnetism, Journal of Magnetism and Magnetic Materials, 54, 1377-1380, (1986).

[18] Baroni, S., Dal Corso, A., De Gironcoli, S., Giannozzi, P., Cavazzoni, C., Ballabio, G., Scandolo, S., Chiarotti, G., Focher, P. and Pasquarello, A., Quantum espresso: opensource package for research in electronic structure, simulation, and optimization, Code available from http://quantum-espresso.org, (2005).

[19] Perdew, J.P., Burke, K. and Ernzerhof, M., Generalized gradient approximation made simple, Physical Review Letters, 77, 38653868, (1996). 
[20] Methfessel, M. and Paxton, A.T., Highprecision sampling for brillouin-zone integration in metals, Physical Review B, 40, 3616-3621, (1989).

[21] Baroni, S., De Gironcoli, S., Dal Corso, A. and Giannozzi, P., Phonons and related crystal properties from Density-Functional Perturbation Theory, Reviews of Modern Physics, 73, 515-562, (2001).

[22] Baroni, S., Giannozzi, P. and Testa, A., Green's-function approach to linear response in solids, Physical Review Letters, 58, 18611864, (1987).
[23] Murnaghan, F.D., The compressibility of media under extreme pressures, Proceedings of the National Academy of Sciences of the United States of America, 30, 244-247, (1944).

[24] Born, M. and Huang, K., Theory of Crystal Lattices, Clarendon, Oxford, (1956).

[25] Pugh, S.F., XCII. Relations between the elastic moduli and the plastic properties of polycrystalline pure metals, Philosophical Magazine and Journal of Science, 45, 823843, (1954). 